\title{
Komplementärmedizin in der ärztlichen Praxis: Mehr als ein zweites „finanzielles Standbein“?
}

\section{Autor: Gerd W. Zimmermann}

\section{ZUSAMMENFASSUNG}

Die Komplementärmedizin subsumiert Diagnose- oder Therapieverfahren, die außerhalb der in der Schulmedizin üblichen Methoden stehen. Im Gegensatz zur Alternativmedizin versucht die Komplementärmedizin aber durch gemeinsames Wirken mit der Schulmedizin Synergieeffekte zu erreichen. Die Leistungen werden deshalb in der Regel von Ärzten erbracht, die auch über die notwendigen schulmedizinischen Kenntnisse verfügen, um diese Leistungen zum Wohle des Patienten einzusetzen.

Im Beitrag werden die Abrechnungsmöglichkeiten für komplementärmedizinische Leistungen erläutert und Beispiele gegeben.

Schlüsselwörter

Komplementärmedizin, GOÄ, Abrechnung, Erstattung

\section{ABSTRACT}

Complementary medicine subsumes diagnostic or therapeutic procedures that are outside the usual methods of conventional medicine. In contrast to alternative medicine, complementary medicine tries to achieve synergy effects by cooperating actively with conventional medicine. The services are therefore usually provided by physicians who also have the necessary knowledge of conventional medicine to use these services for the benefit of the patient.

The article explains the billing options for services in complementary medicine and gives examples.

Keywords

Complementary medicine, GOÄ (scale of fees for physicians), billing, reimbursement

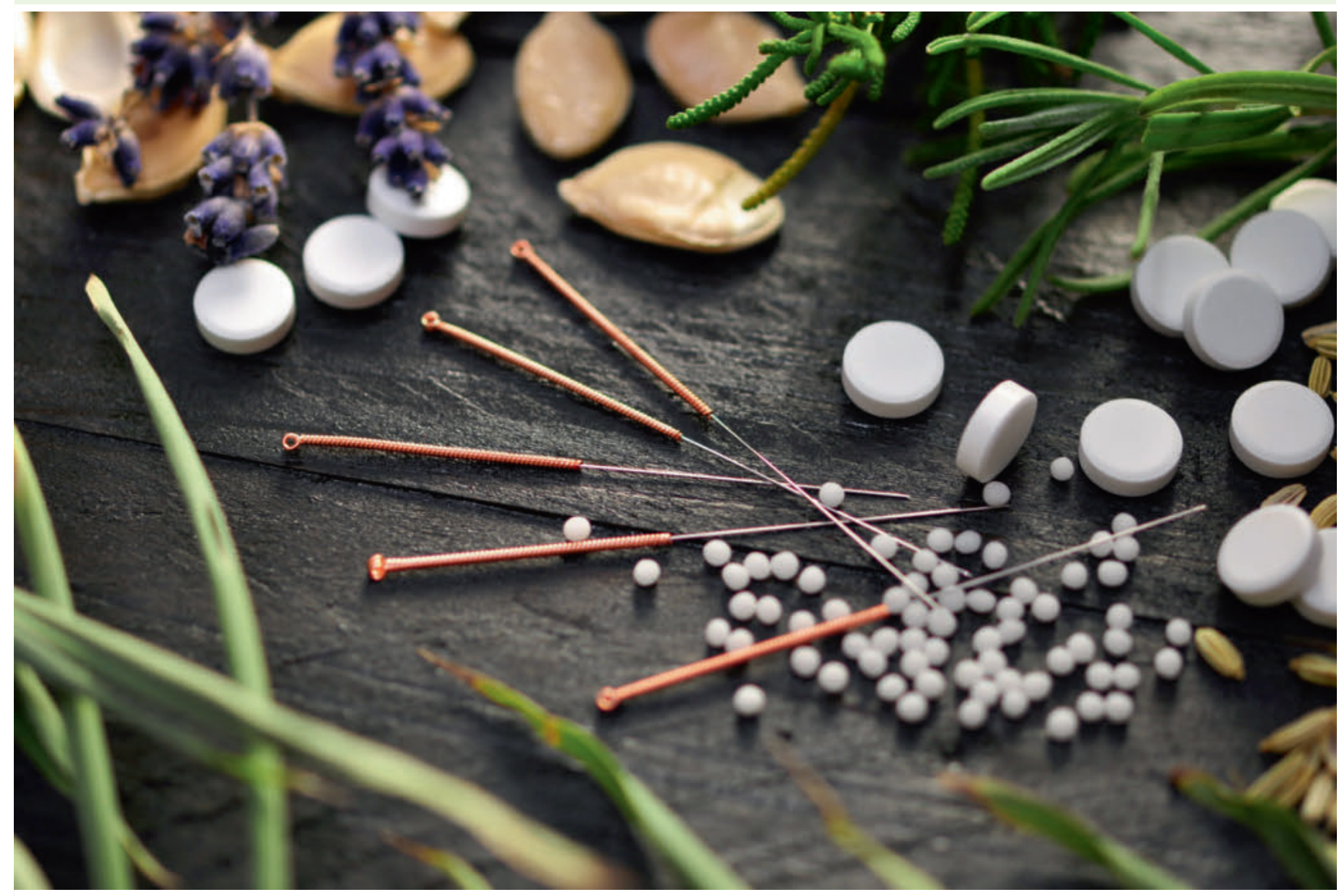

Abb. 1 photocrew/Adobe Stock 
> Tab. 1 Leistungsbeschreibung der GOÄ-Nummern 30 und 31.

GOÄ

Leistungsbeschreibung

Erhebung der homöopathischen Erstanamnese mit einer Mindestdauer von einer Stunde nach biographischen und homöopathisch-individuellen Gesichtspunkten mit schriftlicher Aufzeichnung zur Einleitung einer homöopathischen Behandlung - einschließlich homöopathischer Repertorisation und Gewichtung der charakteristischen psychischen, allgemeinen und lokalen Zeichen und Symptome des jeweiligen Krankheitsfalls, unter Berücksichtigung der Modalitäten, Alternanzen, Kausal- und Begleitsymptome, zur Auffindung des homöopathischen Einzelmittels, einschließlich Anwendung und Auswertung standardisierter Fragebogen

31 Homöopathische Folgeanamnese mit einer Mindestdauer von 30 Minuten unter laufender Behandlung nach den Regeln der Einzelmittelhomöopathie zur Beurteilung des Verlaufs und Feststellung des weiteren Vorgehens - einschließlich schriftlicher Aufzeichnungen
Euro

$120,66^{*}$

$60,33^{*}$

*Beim Honorar ist jeweils der für die einzelne Leistung gültige Schwellenwert zugrunde gelegt.

Komplementärmedizinische Leistungen werden von einigen gesetzlichen und in Abhängigkeit vom Vertragsinhalt auch privaten Krankenkassen bezahlt. Die Abrechnung erfolgt deshalb entweder auf der Grundlage von Sonderverträgen, in der Mehrzahl aber nach den in der Gebührenordnung für Ärzte (GOÄ) zugrunde gelegten Gebührenordnungspositionen. Aber auch hier übernehmen einige GKV-Kassen die Kosten im Erstattungsverfahren zumindest teilweise.

\section{Die wichtigsten diagnos- tischen Leistungen in der Komplementärmedizin}

Die einzelnen GOÄ-Abrechnungspositionen sind im Moment noch über das ganze Gebührenordnungsverzeichnis verteilt und müssen zusammengesucht werden. In der z. Zt. beratenen neuen GOÄ erhält die Komplementärmedizin einen eigenen Abschnitt.

Einstiegsleistung bei der Komplementärmedizin ist in der Regel die ausführliche Diagnostik im Rahmen der Erstund Folgeanamneseerhebung nach den Nrn. 30 und 31 GOÄ ( $\triangleright$ Tab. 1). Die Leistung nach Nr. 30 ist innerhalb von einem (Kalender-)Jahr nur einmal berechnungsfähig, die nach Nr. 31 innerhalb von sechs Monaten höchstens dreimal. Neben der Nr. 31 GOÄ können in gleicher Sitzung die GOÄ-Nrn. 1, 2, 3, 4 und 34, neben der Nr. 30 GOÄ zusätzlich die Nrn. 804, 806, 807, 808, 812, 817, 835, 849, 861, $862,863,864,870,871,886$ und 887 nicht berechnet werden. Dauert die Erhebung einer homöopathischen Erstanamnese bei einem Kind bis zum vollendeten 14. Lebensjahr weniger als eine Stunde, mindestens aber eine halbe Stunde, kann die Leistung bei entsprechender Begründung mit der Hälfte der Gebühr berechnet werden.

Da die Leistungen im Legendentext ausdrücklich auf die homöopathische Anamnese abzielen, taucht die Frage auf, ob eine Abrechnung auch bei anderen komplemen- tärmedizinischen oder sogar schulmedizinischen Anamneseformen analog möglich ist. Nach einem Urteil des Amtsgerichts Kiel (Az.: 115 C 469/14) wurde dies für die Erhebung einer schmerztherapeutischen Erstanamnese bei chronisch Schmerzkranken durch entsprechend qualifizierte Ärzte bereits bestätigt. Bei der Neufassung der GOÄ wird klargestellt, dass jede Form der (ausführlichen) Form wie z. B. die umweltmedizinische, allergologische, schmerztherapeutische, homöopathische, anthroposophisch-medizinische, naturheilkundliche, traditionell-chinesische und osteopathische Anamnese/Folgeanamnese berechnungsfähig ist.

\section{Weitere diagnostische Verfahren:}

Es ist schwierig, die einzelnen komplementärmedizinischen Leistungen in der GOÄ darzustellen. Da ein Abweichen von der Gebührenordnung gesetzlich ausgeschlossen ist, kommt nur die Möglichkeit der analogen Bewertung nach § 6 Absatz 2 der GOÄ in Betracht. In > Tab. 2 sind zunächst Leistungen auf dieser Grundlage mit entsprechender Bewertung aufgeführt, für die es diesbezüglich Vorschläge gibt:

Weitere diagnostische Verfahren wie die „AyurvedaMaharishi-Therapie“, die „Pulsbestimmte Ausleitung mit Bioresonanztherapie, auch im Sinne der Isopathie, Autonosode“, die „Erfassung der Mundbatteriemessung“, die „Durchführung von Testverfahren der psychophysiologischen Regulationstherapie im Sinne der Lüscher Color Diagnostik (LCD)“ oder der „Umfangreiche Arzneimittel-Test nach Übersichtsmessungen der peripheren Messpunkte“ sind in der aktuellen GOÄ nur indirekt über die allgemeinen Untersuchungsleistungen im Kapitel B und den Fachkapiteln zuzuordnen. In der neuen GOÄ sind alle genannten Leistungen hingegen mit eigenen Abrechnungspositionen vertreten. 
\Tab.2 Analoge Bewertung nach §6 Absatz 2 der GOÄ für komplementärmedizinische Leistungen.

\begin{tabular}{|c|c|c|c|}
\hline GOÄ & Legende & Euro & Kommentierung \\
\hline A651 & $\begin{array}{l}\text { Herzfrequenzvariabilitäts-(HFV-)Messung } \\
\text { zur Messung der Herzrhythmusstabilität } \\
\text { unter sympathischer und parasympathischer } \\
\text { Steuerung, vier Ableitungen, Aufzeichnung } \\
\text { über } 15 \text { Minuten einschließlich Auswertung }\end{array}$ & $26,55^{*}$ & $\begin{array}{l}\text { Das Ruhe-EKG kann nur nach dem 1,8-fachen Satz berechnet } \\
\text { werden. Der hier verwendete analoge Ansatz erlaubt ggf. einen } \\
\text { höheren Multiplikator. }\end{array}$ \\
\hline A838 & Regulationstest, je Sitzung & $73,74^{*}$ & $\begin{array}{l}\text { Gemeint sind hier u. a. der autonome Regulationstest (ART) und } \\
\text { die Psychokinesiologie nach Dr. Klinghardt. }\end{array}$ \\
\hline A838 & $\begin{array}{l}\text { Messung der Leitwertveränderung auf } \\
\text { der Haut über verschiedene Verfahren } \\
\text { (z. B. BFD, BIT) }\end{array}$ & $73,74^{*}$ & $\begin{array}{l}\text { Bei der Ausrichtung der Diagnostik auf bestimmte Körperstruktu- } \\
\text { ren können ggf. andere Analogleistungen wie } 827 \text { (Nervensystem), } \\
839 \text { (Muskel, Bindegewebe), } 651 \text { (Herz-/Kreislaufsystem) oder } 624 \\
\text { (Darm) zum Ansatz kommen. }\end{array}$ \\
\hline A842 & Bioresonanzdiagnostik & $67,02^{*}$ & \\
\hline
\end{tabular}

\Tab.3 Konkrete Abrechnungspositionen für komplementärmedizinische Therapieformen.

\begin{tabular}{|c|c|c|c|}
\hline GOÄ & Legende & Euro & Kommentierung \\
\hline A533 & Colon-Hydro-Therapie & $15,73^{*}$ & \\
\hline A555 & Magnetfeldtherapie & $12,58^{*}$ & $\begin{array}{l}\text { Eine wissenschaftliche Anerkennung der Methode liegt nur für die } \\
\text { Behandlung der atrophen Pseudarthrose sowie bei Endoprothesen- } \\
\text { lockerung, idiopathischer Hüftnekrose und verzögerter Knochen- } \\
\text { bruchheilung, wenn sie in Verbindung mit einer sachgerechten } \\
\text { chirurgischen Therapie durchgeführt wird, vor. }\end{array}$ \\
\hline 747 & $\begin{array}{l}\text { Setzen von Schröpfköpfen, Blutegeln oder die } \\
\text { Anwendung von Saugapparaten }\end{array}$ & $5,89^{*}$ & \\
\hline 285 & Mikroaderlass & $14,74^{*}$ & $\begin{array}{l}\text { Die Leistung beinhaltet eine Entnahme von mindestens } 200 \mathrm{ml} \text {. Bei } \\
\text { weniger als } 200 \mathrm{ml} \text { Blut kann nur die Nr. } 250 \text { berechnet werden. }\end{array}$ \\
\hline A838 & Bioresonanztherapie & $73,74^{*}$ & $\begin{array}{l}\text { Auch die Mora-Therapie und andere vergleichbare Verfahren } \\
\text { können nach dieser Leistung berechnet werden. }\end{array}$ \\
\hline
\end{tabular}

\section{Komplementärmedizinische Therapieformen nach GOÄ}

Auch hier sind in $\triangleright$ Tab. 3 zunächst Leistungen aufgeführt, für die es Vorschläge für eine analoge Bewertung oder sogar konkrete Abrechnungspositionen gibt. Bei der Bewertung der hier dargestellten Leistungen ist jeweils der in der GOÄ vorgesehene Schwellenwert von 1,8 bzw. 2,3 zugrunde gelegt.

Mikroaderlässe können an Akupunkturpunkten oder Reflexzonen vorgenommen werden. Eine zusätzliche Berechnung der Nr. 269 GOÄ (Akupunktur (Nadelstich-Technik) zur Behandlung von Schmerzen, je Sitzung - 26,82 Euro bei 2,3-fachem Satz) wäre deshalb möglich. Üblich ist die Entnahme von $100-500 \mathrm{ml}$.
Die „Pulsierende Signaltherapie“ kann analog nach Nr. 555 GOÄ (12,58 Euro bei 2,3-fachem Satz) berechnet werden. Bei der Matrix-Regenerationstherapie handelt es sich um eine Kombination aus pulsgesteuerter petechialer Saugmassage, modulierter Gleichstromtherapie, Rotlichtbestrahlung, pulsierender Magnetfeldtherapie und einer Biofeedbackbehandlung. Die Berechnung setzt sich somit aus einzelnen, in > Tab. 4 zusammengestellten Elementen zusammen.

\section{Besonderheiten bei der Hyperthermie- behandlung}

In der aktuellen GOÄ gibt es nur eine Empfehlung zur Berechnung der Hyperthermiebehandlung der Prostata bei der Behandlung von Krebserkrankungen nach Nr. 5836 (analog Bestrahlung mittels Beschleuniger mit bis zu zwei 
Strahleneintrittsfeldern - gegebenenfalls unter Anwendung von vorgefertigten, wiederverwendbaren Ausblendungen, je Fraktion - 104,92 Euro).

In der Komplementärmedizin kommt diese Therapieform aber in den folgenden Formen zur Anwendung:

- Ganzkörperhyperthermie (GKHT) durch Behandlung des ganzen Körpers mit Infrarotstrahlung als milde Form (Zieltemperatur bis zu 38,4 Grad C, Dauer 60-90 Minuten),

- Ganzkörperhyperthermie (GKHT) durch Behandlung des ganzen Körpers mit Infrarotstrahlung als moderate Form (Zieltemperatur 38,5-40,5 Grad C, Dauer 2-6h),

- Ganzkörperhyperthermie (GKHT) durch Behandlung des ganzen Körpers mit Infrarotstrahlung als extreme Form (Zieltemperatur von mindestens 41,5 Grad C bis über 42 Grad C, Dauer 4-8 h, ohne Stoffwechselbeeinflussung unter intensivmedizinischer Betreuung),

- Lokale Hyperthermie durch Behandlung mit InfrarotA-Strahlung,

- Oberflächenhyperthermie als lokale Behandlung mittels kapazitiv gekoppelter Elektroden oder hochfrequenter Radiowellen,

- Lokoregionale Hyperthermie mit umschriebenen elektromagnetischen Feldern mittels kapazitiv gekoppelter Elektroden oder hochfrequenter Radiowellen,

Alle genannten Leistungen sind eigenständig in der neuen, zurzeit als Entwurf vollständig vorliegenden GOÄ aufgeführt. Bis zu deren Inkrafttreten (Ziel 1. Oktober 2020) kommen auch hier nur analoge Leistungsberechnungen in Betracht. Die meisten hier aufgeführten Leistungen können ebenfalls analog nach Nr. 5836 GOÄ berechnet werden, dürften dabei wegen des erheblichen Aufwandes aber der stationären Behandlung vorbehalten sein. Bei der ambulant denkbaren Behandlung als lokale Hyperthermie käme die bereits erwähnte Leistung nach Nr. 538 (Infrarotbehandlung, je Sitzung - 4,19 Euro) in Betracht. Die für den auch dort notwendigen Aufwand viel zu geringe Vergütung kann über die Beratungs- und Untersuchungsleistungen des Kapitel B, ggf. unter Verwendung eines höheren Multiplikators ausgeglichen werden.

\section{Weitere therapeutische Verfahren der Komplementärmedizin}

Eine Reihe komplementärmedizinischer Leistungen ist eher typisch für die Praxis, auch dort, wo kein solcher Tätigkeitsschwerpunkt vorliegt.

\section{Eigenblutbehandlung}

Die häufigste diesbezügliche Leistung ist die Eigenblutbehandlung. Die Leistung kann nach Nr. 284 GOÄ berechnet werden (12,08 Euro bei 2,3-fachem Satz) und umfasst die venöse Blutentnahme und die anschließend zumeist intra- 
\ab.4 Abrechnungsbeispiele für „Pulsierende Signaltherapie“ und Matrix-Regenerationstherapie.

\begin{tabular}{|c|c|c|}
\hline Legende & GOÄ & Euro \\
\hline Anwendung von Saugapparaten, je Sitzung & 747 & $5,89^{*}$ \\
\hline $\begin{array}{l}\text { Reizstrombehandlung (Anwendung niederfrequenter Ströme) - auch bei wechselweiser Anwendung } \\
\text { verschiedener Impuls- oder Stromformen und gegebenenfalls unter Anwendung von Saugelektroden } \\
\text { Hinweis: } \\
\text { Wird Reizstrombehandlung nach Nr. } 551 \text { in gleicher Sitzung neben Nr. } 747 \text { an demselben Körperteil oder } \\
\text { an denselben Körperteilen verabreicht, so ist nur die höherbewertete Leistung berechnungsfähig; dies gilt } \\
\text { auch bei Verwendung eines Apparatesystems an mehreren Körperteilen. }\end{array}$ & 551 & $5,04^{*}$ \\
\hline Infrarotbehandlung, je Sitzung & 538 & $4,19^{*}$ \\
\hline Pulsierende Magnetfeldtherapie & A555 & $12,58^{*}$ \\
\hline Biofeedbackbehandlung & A838 & $73,74^{*}$ \\
\hline
\end{tabular}

> Tab. 5 Abrechnungsbeispiel für die Ozontherapie.

\begin{tabular}{|c|c|c|}
\hline Legende & GOÄ & Euro \\
\hline Injektion, intravenös & 253 & $9,38^{*}$ \\
\hline $\begin{array}{l}\text { Aderlass aus der Vene oder Arterie mit Entnahme von mindestens } 200 \text { Milliliter Blut - gegebenenfalls } \\
\text { einschließlich Verband }\end{array}$ & 285 & $14,74^{*}$ \\
\hline $\begin{array}{l}\text { Reinfusion der ersten Einheit (mindestens } 200 \text { Milliliter) Eigenblut oder Eigenplasma - einschließlich Identitäts- } \\
\text { sicherung im AB0-System (Bedside-Test) }\end{array}$ & 286 & $29,49^{*}$ \\
\hline $\begin{array}{l}\text { Reinfusion jeder weiteren Einheit (mindestens } 200 \text { Milliliter) Eigenblut oder Eigenplasma im Anschluss an die } \\
\text { Leistung nach der Nummer } 286 \text { - einschließlich Identitätssicherung im AB0-System (Bedside-Test) }\end{array}$ & $286 a$ & $13,41^{*}$ \\
\hline
\end{tabular}

muskulär vorgenommene Reinjektion. Häufig wird das Blut vor der Wiederverabreichung mit einem Ozon-SauerstoffGemisch angereichert. Diese und andere Varianten fallen ebenfalls unter die Nr. 284, können jedoch als Erschwernisse bei der Leistungserbringung über den Gebührenrahmen geltend gemacht werden. Ursprünglich war diese Therapie sogar Bestandteil des EBM, wurde im Rahmen einer Reform aber abgeschafft. Kassen vertreten deshalb mitunter die Auffassung, dass es sich hier um keine individuelle Gesundheitsleistung (IGeL) handelt und die Leistung innerhalb der EBM-Pauschalen nach den Nrn. 250 und 252 EBM berechnungsfähig sei. Dafür gibt es allerdings keinerlei verbindlichen Hintergrund.

\section{Ozontherapie}

Die Ozontherapie gehört ebenfalls zu den nicht vertragsärztlich anerkannten Heilmethoden und kann deshalb nur nach GOÄ berechnet werden. Zum Ansatz kommen entsprechend dem Leistungsinhalt die Nr. 253, 285, 286 und 286 a ( $\triangleright$ Tab. 5). Beihilfefähig ist die Leistung nur bei Gasinsufflationen, wenn damit arterielle Verschlusserkrankungen behandelt werden und die Festsetzungsstelle aufgrund des Gutachtens eines von ihr bestimmten Amts- oder Vertrauensarztes die Beihilfefähigkeit vor Beginn der Behandlung anerkannt hat.

Zu den traditionell chinesischen Behandlungsmethoden (TCM) gehört insbesondere die Akupunktur. Hier stehen die Leistungen nach den GOÄ-Nrn. 269 (Akupunktur (Nadelstich-Technik) zur Behandlung von Schmerzen, je Sitzung - 26,82 Euro bei 2,3-fachem Satz) und 269a (Akupunktur (Nadelstich-Technik) mit einer Mindestdauer von 20 Minuten zur Behandlung von Schmerzen, je Sitzung - 46,92 Euro bei 2,3-fachem Satz) zur Verfügung. Nach einem Urteil des Oberlandesgerichts (OLG) Nürnberg (Az.: 1 U 9/08) kann diese Leistung auch für die ImplantatOhr-Akupunktur berechnet werden. Wegen des deutlich höheren Zeitaufwandes gegenüber der Nadelstich-Technik kann hier der Ansatz des 3,5-fachen Gebührensatzes als angemessen angesehen werden. Neben der jeweiligen Gebühr für die Akupunkturleistungen können die Kosten z. B. für die Nadeln gemäß § 10 Abs. 2 Nr. 1 GOÄ zusätzlich in Rechnung gestellt werden. 


\section{Behandlung mittels Vibratode}

Die „Behandlung mittels Vibratode im Sinne einer biomechanischen Muskelstimulation“ kann analog nach Nr. 558 GOÄ (Apparative isokinetische Muskelfunktionstherapie, je Sitzung 12,60 Euro bei 1,8-fachem Satz) berechnet werden.

\section{Tuina}

Eine Berechnung der „Chinesische Manualtherapie Tuina mit mindestens vier Grifftechniken“ kann analog nach Nr. 3306 GOÄ (Chirotherapeutischer Eingriff an der Wirbelsäule, 19,85 Euro bei 2,3-fachem Satz) berechnet werden. Entsprechend einer Abrechnungsempfehlung der Bundesärztekammer kann so auch ein chirotherapeutischer Eingriff an einem oder mehreren Extremitätengelenken, je Sitzung, abgerechnet werden. Eine mehr als zweimalige Berechnung im Behandlungsfall muss allerdings begründet werden. Dies wäre z. B. der Fall, wenn die Eingriffe an der Wirbelsäule und an Gelenken vorgenommen werden.

\section{Spezielle Massageformen}

Die gebührenordnungstechnische Darstellung spezieller Massageformen wie der „Spezialmassage gemäß Ayurveda“ oder der „Rhythmischen Massage nach Ita Wegman“ ist schwierig, da die Bewertung der einfachen Massage in der GOÄ viel zu niedrig angesetzt ist. Da die Ayurveda-Massage mindestens 60 Minuten andauert und die Massage nach Ita Wegman mindestens 20 Minuten, wäre der Ansatz des 3,5-fachen Satzes bei der Nr. 521 GOÄ angemessen. Diese Leistung darf It. GOÄ aber nur bis zum 1,8-fachen Regelsatz gesteigert werden, und der Höchstsatz liegt damit bei dem 2,5-fachen Multiplikator.
> Tab. 6 Gebührenrahmen nach §5 Absatz 1 der GOÄ.

\begin{tabular}{|c|c|c|c|c|}
\hline & \multicolumn{4}{|l|}{ Leistungen } \\
\hline & \multirow[t]{2}{*}{ persönlich } & \multirow{2}{*}{$\begin{array}{l}\text { persönlich } \\
\text { (nach } \\
\S 5 \text { Abs. 5) }\end{array}$} & \multicolumn{2}{|l|}{ technisch } \\
\hline & & & ohne Labor & Labor \\
\hline $\begin{array}{l}\text { Schwellenwert } \\
\text { Höchstsatz }\end{array}$ & $\begin{array}{l}2,3 \\
3,5\end{array}$ & $\begin{array}{l}1,8 \\
2,3\end{array}$ & $\begin{array}{l}1,8 \\
2,5\end{array}$ & $\begin{array}{l}1,15 \\
1,3\end{array}$ \\
\hline $\begin{array}{l}\text { Standardtarif nach } \\
\S 5 \text { b GOÄ }\end{array}$ & 1,8 & 1,8 & 1,38 & 1,16 \\
\hline
\end{tabular}

In diesen Fällen kommt die Möglichkeit einer abweichenden Honorarvereinbarung nach §2 Absatz 1 der GOÄzum Tragen. Mit dem Patienten muss eine Vereinbarung mit einem erhöhten Multiplikator je Leistung oder Leistungsgruppe bekannt gegeben werden. Eine pauschale Regelung ist nicht zulässig. Außerdem müssen dem Patienten die Gebührenordnung oder die infrage kommenden Abschnitte derselben zur Einsicht vorgelegt werden, damit er sich die Höhe seiner Zahlung insgesamt und ggf. des Betrags, den seine Versicherung nicht übernimmt, ausrechnen kann. Der Arzt muss den Patienten ggf. darauf hinweisen, dass er einen bestimmten Differenzbetrag weder von der Beihilfe noch von seiner Privatkrankenkasse ersetzt bekommt. Eine durch die GOÄ (oder anderweitig) festgelegte Höchstgrenze für eine solche Abdingung gibt es dabei nicht. Bedauerlicherweise ist eine solche Abdingung allerdings bei Leistungen des Kapitels E (Physikalische Therapie) und damit bei der Nr. 521 GOÄ ausgeschlossen. In dem Entwurf für die neue, betriebswirtschaftlich kalku- 
lierte GOÄ wird für die Ayurveda-Massage ein Honorar von rund 88 Euro und für die Massage nach Ita Wegman von rund 32,50 Euro festgelegt. Ein solches Ergebnis könnte bei der Massage nach Ita Wegman durch den 3,5-fachen Satz bei der Nr. 3 GOÄ (Eingehende, das gewöhnliche Maß übersteigende Beratung, mindestens 10 Minuten - 30,59Euro) und bei der Ayurveda-Massage mit der Kombination aus den GOÄ-Nrn. 3 und 5 (Symptombezogene Untersuchung) bei einem Multiplikator zwischen 6 und 7 erreicht werden.

\section{Besondere Behandlungstechniken}

Bestandteil der Komplementärmedizin sind auch besondere Behandlungstechniken wie die „Atemtherapie nach Middendorf oder vergleichbare Verfahren“ bzw. die „Körperarbeit mittels Alexander-Technik“. Nach der erwähnten betriebswirtschaftlichen Kalkulation auch dieser Leistungen müsste ein Honorar von 42 Euro bzw. 21,35 Euro resultieren. Auch hier kommen deshalb vorhandene GOÄLeistungen praktisch nicht in Betracht, da sie viel zu niedrig bewertet sind. Der Ansatz der Nr. 505 GOÄ würde selbst beim 2,5-fachen Satz nur zu einem Honorar von 12,38 Euro führen. Eine Abdingung als Ansatz der Nr. 3 GOÄ zum 5-fachen Satz führt hingegen zu einem angemessenen Honorar von 43 Euro.

Die Alexander-Technik ist eine Methode des Lernens, die es möglich macht, sich bewusster mit dem Körper zu befassen und so zu einem guten Körpergebrauch zu gelangen. Auch diese Leistung findet in der aktuellen GOÄ keine angemessen vergütete Abrechnungsposition, sodass mit Blick auf das o. g. kalkulierte Honorar auch hier der Ansatz der Nr. 3 GOÄ, allerdings nur mit einem Multiplikator von 2,5, der wiederum keine Abdingung erforderlich macht, zum Ansatz kommen könnte.

Beachtenswert ist dabei, dass die Gebührenordnung beim Ansatz der Nr. 3 GOÄ bei einer mehr als einmaligen Berechnung im Behandlungsfall eine besondere Begründung fordert. Dies könnte in diesem Fall der Hinweis auf die zeitaufwendige Behandlungsmethode sein.

\section{Was muss bei einer Abdingung beachtet werden?}

Eine zulässige Abdingung ist dann erforderlich, wenn man beabsichtigt, die innerhalb des Gebührenrahmens in $\S 5$ festgelegten Gebühren zu überschreiten oder innerhalb des Gebührenrahmens eine Gebührenhöhe zu wählen, die sich aus den Bemessungsfaktoren nach $\S 5$ nicht ergeben würde. Dazu ist das Einvernehmen mit dem Patienten vor Beginn der Behandlung herzustellen. Man muss, wenn man eine Abdingung anstrebt, auch die gültige Berufsordnung der zuständigen Landesärztekammer beachten. Nach § 12 Abs. 1 Satz 1 der (Muster-)Berufsordnung des Deutschen Ärztetages muss eine Honorarforderung „angemessen sein “. Hier ist die erwähnte betriebswirtschaftliche Kalkulation bei den Leistungen der neuen GOÄ sehr hilfreich. Selbst wenn es nicht in ansehbarer Zeit zu einem Inkrafttreten dieser GOÄ-Reform kommen sollte, stehen diese, auch mit den Privatkassen vereinbarten „Preise“ im Raum und können als Grundlage für analoge Bewertungen nach $\S 6$ Absatz 2 und Abdingungen nach $\S 2$ Absatz 1 der alten GOÄ herangezogen werden.

Ein Beispiel für eine rechtskonforme Umsetzung der Abdingungsvoraussetzungen ist im Anhang (S. 264) angefügt.

\section{Möglichkeiten der Honorargestaltung}

Die meisten Leistungen in der GOÄ erlauben eine Gestaltung des angegebenen Honorars für die einzelne Leistung vom Einfachen bis zum Dreieinhalbfachen. Innerhalb dieses Rahmens gibt es eine Begründungsschwelle in Höhe des zweieinhalbfachen Satzes. Hier handelt es sich um die sogenannten „persönlichen“ Leistungen. Für sogenannte „technische“ Leistungen gibt es zwei unterschiedliche niedrigere Gebührenspannen ( Tab.6). Daneben gibt es noch Abrechnungspositionen, die nur ohne jegliche Multiplikation, also mit dem Einfachsatz oder den tatsächlichen Kosten berechnet werden dürfen, so die Zuschläge A, B, C, $D, E, F, G, H, J, K 1, K 2$ und die Wegegelder und Reiseentschädigungen sowie die Nrn. 52, 95, 96.

Seit 1982 ist die Vertragsfreiheit zwischen Arzt und Patient im privatärztlichen Behandlungsverhältnis auf die in § 2 der GOÄ zusammengefassten Bestimmungen eingeschränkt. Beim Abschluss einer abweichenden Honorarvereinbarung muss die GOÄ für privatärztliche Leistungen in jedem Fall Grundlage bleiben. Unter verbindlicher Bezugnahme auf eine bestimmte Leistung bzw. Gebührenposition in der GOÄ kann nur der Steigerungssatz, nicht aber die Punktzahl für die Leistung oder ein abweichender Punktwert vereinbart werden. Eine Vereinbarung von Pauschalhonoraren ist ebenfalls nicht zulässig.

Eine abweichende Honorarvereinbarung muss darüber hinaus vor Erbringung der Leistung erfolgen und aus Gründen der Rechtsklarheit und -sicherheit in einem gesonderten Schriftstück festgehalten werden, das außer den in §2 Absatz 2 GOÄ genannten Inhalten keine weiteren Erklärungen enthalten darf. Gemäß § 2 Absatz 2 GOÄ muss der Patient darüber aufgeklärt werden, dass im Falle einer abweichenden Honorarvereinbarung die Kostenerstattung durch eine Krankenkasse möglicherweise nicht in vollem Umfang gewährleistet ist. Die abweichende Honorarvereinbarung setzt „eine persönliche Absprache im Einzelfall“ voraus. Der Patient muss in einem persönlichen Gespräch mit dem Arzt über die Modalitäten der Behandlung und der Vergütung informiert werden.

In allen Fällen können entstehende Kosten zusätzlich, aber auch nur auf der Grundlage der GOÄ ( $§ 10$ Ersatz von Auslagen) berechnet werden. 
HONORARGESTALTUNGSMÖGLICHKEITEN: ZWEI FALLBEISPIELE

> Tab. 7 35-jähriger Patient mit Reizdarmsyndrom (Diagnostik und Therapie).

\begin{tabular}{|c|c|c|c|c|c|}
\hline \multirow[b]{2}{*}{ Leistung } & \multirow{2}{*}{ GOÄ } & \multicolumn{4}{|c|}{ Euro nach GOÄ-Faktor } \\
\hline & & $2,3 / 1,8$ & $3,5 / 2,5$ & Abdingung & Multiplikator \\
\hline Eingehende Anamneseerhebung & 30 & 120,66 & 183,61 & 200 Euro & 3,81 \\
\hline Ganzkörperuntersuchung & 8 & 34,85 & 53,02 & 80 Euro & 5,28 \\
\hline Rektale Untersuchung & 11 & 8,05 & 12,25 & 15 Euro & 4,29 \\
\hline Colon-Hydro-Therapie* & A533 & 15,73 & 21,85 & $\begin{array}{l}50 \text { Euro } \\
100 \text { Euro }\end{array}$ & $\begin{array}{l}5,72 \\
11,44\end{array}$ \\
\hline \multicolumn{6}{|l|}{ ggf. zusätzliche Kosten nach §10 GOÄ } \\
\hline \multicolumn{6}{|c|}{ *Der Schwellensatz liegt hier bei 1,8 und der Höchstsatz bei 2,5. } \\
\hline \multicolumn{6}{|c|}{ - Tab. 8 18-jährige Patientin mit Neurodermitis und Migräne (nur Diagnostik). } \\
\hline & \multicolumn{5}{|c|}{ Euro nach GOÄ-Faktor } \\
\hline Leistung & GOÄ & 2,3 & 3,5 & Abdingung & Multiplikator \\
\hline Beratung & 1 & 10,72 & 16,31 & 25Euro & 5,36 \\
\hline Untersuchung der Haut & 7 & 21,46 & 32,66 & 40 Euro & 4,29 \\
\hline Neurologische Untersuchung & 800 & 26,15 & 39,80 & 50 Euro & 4,40 \\
\hline $\begin{array}{l}\text { Messung der Leitwertveränderung auf der Haut } \\
\text { (BFD) }\end{array}$ & A838 & 73,74 & 112,21 & 120 Euro & 3,74 \\
\hline Bioresonanzdiagnostik & A842 & 67,02 & 101,99 & 100 Euro & 3,43 \\
\hline
\end{tabular}

\section{Interessenkonflikt}

Der Autor erklärt, dass keine wirtschaftlichen oder persönlichen Verbindungen bestehen.

\section{Autor}

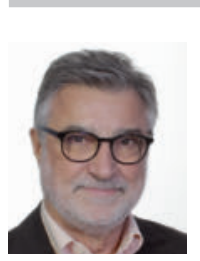

\section{Gerd Zimmermann}

ist Allgemeinarzt und seit 1979 in hausärztlicher Gemeinschaftspraxis in Hofheim/Taunus niedergelassen. 1991-2007 Mitglied des Bundesvorstands des Deutschen Hausärzteverbands, zuletzt stv. Bundesvorsitzender; seit 2011 Sachverständiger des Bundesverbands für die Verbandszeitschrift „Der Hausarzt“. Zahlreiche weitere Ämter in Gremien und Verbänden, u. a. 2005-2012 stv. Vorsitzender des hauptamtlichen Vorstands der Kassenärztlichen Vereinigung Hessen.

\section{Korrespondenzadresse}

\section{Dr. med. Gerd W. Zimmermann}

Facharzt für Allgemeinmedizin

Psychosomatische Grundversorgung, Diabetologie

Am Untertor 4

65719 Hofheim am Taunus

E-Mail: info@gwzimmermann.de

\section{Bibliografie}

DOI https://doi.org/10.1055/a-1016-1425

EHK 2019; 68: 256-264

(c) MVS Medizinverlage Stuttgart GmbH \& Co. KG ISSN 0014-0082 


\section{Erklärung über die Inanspruchnahme einer Privatbehandlung}

\section{Herr/Frau}

\begin{tabular}{|l|l|l|}
\hline Name & Vorname & Geburtsdatum \\
\hline & & \\
\hline Anschrift & & \\
\hline & \\
\hline
\end{tabular}

\section{Zwischen}

als behandelndem Arzt und dem o.g. Zahlungspflichtigen wird in persönlicher Absprache nach § 2 der Gebührenordnung für Ärzte (GOÄ) durch die Unterzeichnenden folgende Honorarvereinbarung geschlossen:

Beschreibung

Unterschrift

Ich verzichte bei den unten näher definierten Leistungen auf die gesetzlichen Leistungen (Sachleistungen) und werde die entsprechenden Kosten nach der zurzeit gültigen GOÄ (Gebührenordnung für Ärzte) selbst tragen.

Im Behandlungszeitraum vom ............./20___ bis ................/20___ verzichte ich auf die gesetzlichen Leistungen (Sachleistungen) und werde die entsprechenden Kosten nach der zurzeit gültigen GOÄ (Gebührenordnung für Ärzte) selbst tragen.

Sofern es sich bei den unten näher definierten Leistungen um solche handelt, die nicht Bestandteil des GKV-Kataloges sind, ist mir bewusst, dass ich gegenüber meiner Krankenkasse keinen Erstattungsanspruch habe!

\begin{tabular}{|l|l|l|l|l|}
\hline Datum & GOÄ-Nr. & Faktor & Erläuterung & Betrag \\
\hline & & & & \\
\hline & & & & \\
\hline & & & & \\
\hline & & & & \\
\hline \\
\hline
\end{tabular}

Ort/Datum

\begin{tabular}{l|} 
Unterschrift Patient \\
\hline Unterschrift Arzt/Ärztin
\end{tabular}

